\title{
Knowledge and Practices of Mothers about Diarrhea in Children Under Three Years Old in Heevi Pediatric Teaching Hospital in Duhok City
}

\author{
Omed S. Saadallah" ${ }^{*}$, Gulistan A. Saido² \\ ${ }^{1}$ Department of Fundamentals and Adult Nursing, College of Nursing, University of Duhok, Duhok, Kurdistan Region, Iraq, ${ }^{2}$ Department of \\ Medical Education Development, College of Medicine, University of Duhok, Duhok, Kurdistan Region, Iraq
}

\section{*Corresponding author: Omed S. Saadallah, Department of Fundamentals and Adult Nursing, College of Nursing, University of Duhok, Duhok, Kurdistan Region, Iraq. \\ E-mail: omed.saadallah@ uod.ac}

Received: 10 June 2020

Accepted: 23 August 2020

Published: 30 June 2021

DOI

10.25156/pti.v11n1y2021.pp52-57

\section{A B S T R A C T}

Background and Objectives: Diarrheal disease is the second important reason of death in children under 5 years old and is responsible for killing around 760,000 children every year. Diarrhea can last for several days and it can leave the body without the water and salts that are necessary for survival. Most people who die from diarrhea actually die from severe dehydration and fluid loss. The aim of this study is to assess the relationship between mother's knowledge and their practice about diarrhea in children $<3$ years old. Research Methodology: A cross-sectional study was conducted on 50 children with diarrheal episodes attending inpatient word. A set of questionnaires from previous studies was adopted and modified to assess the relationship between mother's knowledge and their practice about diarrhea in children less than 3 years old in Heevi Pediatric Teaching Hospital in Duhok City. Results: More than half of participants (68\%) had poor knowledge, though; the $32 \%$ had good knowledge. In terms of mother's practice, most of the participants $(64 \%)$ had poor mother practice; whereas, only $36 \%$ were good mother practice. However, there was a negative correlation between mother knowledge and mother practice $(P \leq 0.001)$. Conclusions: The findings of the study indicate that due to lack of knowledge and poor practice about management of diarrhea the children were suffering from many complications. Relevance to clinical practice: Nurse educators have a significant role in providing health education to mothers about diarrhea in children.

Keywords: Diarrhea; Mother knowledge; Mother practice; Oral rehydration solution

\section{INTRODUCTION}

As in the topics, diarrheal disease is the second important cause of death in children $<5$ years old, and it is responsible for killing about 760,000 children each year(Al-Hindi, 2017). Diarrhea a take more than a few days, and can leave the body without the water and salts that are essential for survival. Many people who die because of diarrhea actually die from severe dehydration and fluid loss (Hamed, 2016). Children who are underweight or have weakened immunity as well as people living with HIV are most at risk of severe diarrhea. Diarrhea is typically a symptom of an infection in the intestinal tract, which can be caused by a variety of bacterial, viral, and parasitic organisms (Shane et al., 2017).

Infection is spread through contaminated food or drinking water, or from person-to-person because of poor hygiene (Teshale et al., 2010). Diarrhea is the condition of having at least three loose liquid bowel movements each day (Spika et al., 1986). It often lasts for a few days and can result in dehydration due to excessive fluid loss with appear signs and symptoms of dehydration often begin with loss of the normal stretchiness of the skin and irritable behavior. This can growth to decreased urination, a fast heart rate, and loss of skin color, reduction in responsiveness as it becomes more severe. Loose but non-watery stools in babies who are breastfeed, however, may be normal patient (Hussein, 2018). Children who have impaired immunity or underweight or as well as people living with HIV are mostly at risk of life-threatening diarrhea (Islam et al., 2018).

Diarrhea is the second important cause of child morbidity and mortality, particularly in the developing countries. It is estimated that there were 2.5 billion episodes and 1.5 million deaths annually in children $<5$ years (Agegnehu et al., 2019). Diarrhea is not lethal itself, but the improper knowledge of mother and their misdirected approach toward its management leads to high degree of mismanagement and result in severe dehydration (Asghar et al., 2016).

The World Health Organization (WHO) diarrhea is defined as the passageway of three or more loose or liquid stools 
per day (or more frequent passage than is normal rate for the individual) (Blaabjerg et al., 2017). Interventions to avoid diarrhea, such as safe drinking water, hand washing with soap, and use of improved sanitation could help to reduce disease risk. Diarrhea can treat with a solution of clean water salt, sugar, and with zinc tablets (Ahs et al., 2010). There are three clinical types of diarrhea: First; acute watery diarrhea - lasts several hours or days, and includes cholera; second; acute bloody diarrhea - also called dysentery; and third; persistent diarrhea - pasts 14 days or longer (Matsebula-Myeni, 2019).

The Scope of diarrheal disease; diarrheal disease is a leading cause of child mortality and morbidity in the world, and mostly results from contaminated food and water sources (Al-Hindi, 2017). Worldwide, 780 million individuals lack access to improved drinking water and 2.5 billion lack-improved sanitation. Diarrhea due to infection is widespread throughout developing countries (Singh et al., 2019). In developing countries, children under 3 years old experience on average three episodes of diarrhea every year (Mahor, 2013). Each episode deprives the child of the nutrition necessary for growth.

As a result, diarrhea is a major cause of malnutrition, and malnourished children are more likely to fall ill from diarrhea. The most severe threat posed by diarrhea is dehydration. During a diarrheal episode, water and electrolytes (sodium, chloride, potassium, and bicarbonate) are lost through liquid stools, vomit, sweat, urine, and breathing (Chandra, 2012). Dehydration occurs when these losses are not replaced. The degree of dehydration is rated on a scale of three early dehydration - no signs or symptoms.

\section{Moderate Dehydration}

Start with Thirst, Restless, or irritable behavior, decreased skin elasticity and sunken eyes, finally severe dehydration: Symptoms become more severe; shock, with diminished consciousness, lack of urine output, cool, moist extremities, a rapid and weak pulse, low or undetectable blood pressure, and pale skin. Death can follow severe dehydration if body fluids and electrolytes are not replenished, either with oral rehydration salts (Ahs et al., 2010) solution, or through an intravenous drip (Banik, 2013).

\section{The Main Causes of diarrhea; Infection}

Diarrhea is a symptom of infections began by a host of bacterial, viral, and parasitic organisms, most of which are spread by feces-contaminated water (Di Genova and Tonelli, 2016). Infection is more common when there is a shortage of adequate sanitation and hygiene, cooking, cleaning, and safe water for drinking. Rotavirus and Escherichia coli are the two most common etiological agents of diarrhea in developing countries. Next causes are malnutrition: Children who die from diarrhea often suffer from underlying malnutrition, which makes them more vulnerable to diarrhea. Every diarrheal episode, in turn, makes their malnutrition even worse (O'Ryan et al., 2005).

Diarrhea is a leading reason of malnutrition in children $<5$ years old. Third causes are source: Water contaminated with human feces, for example, from sewage, septic tanks, and latrines, is certain concern. Animal feces also contain microorganisms that may cause diarrhea (Smith and Fratamico, 1995). Finally, the other causes are diarrhea disease can also spread from person-to-person, aggravated by poor personal hygiene. Food is another main cause of diarrhea when it is prepared or stored in unhygienic conditions. Water can contaminate food during irrigation. Fish and seafood from polluted water may also contribute to the disease (Grimason et al., 2014).

The prevention and treatment depend on some key measures to prevent diarrhea include: Access to safe drinking-water; use of improved sanitation; hand washing with soap; exclusive breastfeeding for the first 6 months of life; good personal and food hygiene; health education about how infections spread; and Rotavirus vaccination (WHO, 2015). Diarrhea is the second leading cause of child morbidity and mortality, especially in the developing countries. Diarrhea is the most prevalent pediatric disease and causes of death in children under 5 years-of-age in developing countries. Moreover, diarrheal diseases cause serious economic problems for developing countries (Sumampouw et al., 2019).

In few studies have been recognized and highlighting the effect of diarrhea on child mortality and morbidity among children under 5 years of age. Based on Chavan (2013) acute gastroenteritis remains to be one of the vital causes of childhood morbidity and mortality and is related with an estimated 4 million deaths each year worldwide (Shah et al., 2015). Viral agents account for $75 \%$ of these infections, the major pathogen being rotavirus, which is associated with up to $50 \%$ of diarrheal episodes among infants and young children (Mokomane et al., 2018). A study conducted by Badowski et al, (2011) in Tanzania, identified a number of behavioral practices that may perpetuate the transmission of pathogens through fecal contamination of hands and drinking water (Shah et al., 2015). Researchers further reported that inadequate hand washing was the core causal factor in causing diarrhea because it obviously showed that hand washing has been associated with a reduction of diarrheal disease approximately $42-48 \%$ and $39 \%$ in highincome countries or $32 \%$ in low-income countries. Mahor (2013) advocated that transmission sources of diarrhea are untreated drinking water. 
The population having household sanitation system has less chances of developing diarrheal diseases, another source of diarrhea in children $<5$ years is left over food or spoiled food (Mathur et al., 2019). He also mentioned that other major cause of diarrhea in children is bottle-feeding rather than breastfeeding which increase the risk of diarrhea in children (Suliman, 2017). Melinda and Munos, (2010) stated that that previous studies on rotavirus were but no other reported studies are carried out before a decade ago in the capital, Addis Ababa available in other regions of the country and they had mentioned that rotavirus is the major cause of diarrhea.

The leading cause of death from acute diarrhea is the loss of water and essential minerals, which can be compensated in most cases by an oral rehydration solution (ORS) (Ahs et al., 2010). The mother has an important role to decrease the rate of diarrheal episodes by good management of diarrhea in children and by applying preventive measurement but according to many studies they have poor knowledge and practice about diarrhea in children under three years old in developing countries. The aim of this study is to assess the relationship between knowledge and practice of mothers about diarrhea in children under 3 years old in Heevi Pediatrics Teaching Hospital in Duhok city/Kurdistan region - Iraq.

\section{RESEARCH METHODOLOGY}

\section{Study Design, Setting, PARTICIPANTS, and Procedure} A cross-sectional study, quantitative descriptive design was carried out to assess the mother's knowledge and practice about diarrhea in children under three years old in Heevi Pediatric Teaching Hospital in Duhok City. The Heevi Pediatric Teaching Hospital in Duhok City, which is, consists of many departments such as emergency unit, medical ward, surgical word, laboratory, X-ray, clinical, and consulting units. The hospital receives patients with different health problem such as upper and lower respiratory infection, diarrheal children, shortness of breath, premature baby, and so on.

The target population participating in this study is 50 children with diarrheal inpatients in Heevi Pediatric Teaching Hospital in Duhok city; the mothers were selected from different places, ages and knowledge. The sample size was 50 participants we use SPSS "Statistical Package for the Social Sciences" software Ver.22 to entry and calculate the results of the study, the study period from March 5 to April 5, 2015.The inclusion criteria were the patients under 3 years with diarrhea, both gender and diarrhea more than 2 day. The mothers with psychiatric problems and mother with multiple problems (speaking and hearing) were excluded from study.
In this study, the data were collected using close-ended questions, a set of questionnaires with self-administration of distribution on participants, which was consisted of 27 items that are adapted and modified from a few previous studies on knowledge and practice of mothers about diarrhea in children under 3 and 5 years old. The population involved of all eligible participants from inpatient by appropriate questionnaire taking using convenient sampling. The questionnaire consists of three parts, the first part about socio-demographic characteristics, which was consisted of six items. The second part of questionnaire served to assess mother's knowledge, which is consisted of 13 items in the form of (Yes, No, and Not sure) to get more accurate and clear answer. The third part of questionnaire in this study served to identify the mother practice. This section is consisted of eight items in a Likert scale form, (1, 2, and 3) considered as poor practice while (4 and 5) considered as good practice, therefore; in this study, most of these important factors have been comprised such as age, gender, educational level, employment status, and monthly income.

The dependent variables in this study are the knowledge and practice of mother regarding the management of diarrhea in children under 3 years old, who consisted of 13 items for mother knowledge, and eight items regard to mother practice. Therefore, the total some of actual score in scales would range from 13 to 25 for mother knowledge divided into two groups $(<19)$ are considered poor knowledge and $(>20)$ are considered good knowledge. While the mother practice sum score ranges from 14 to 38 and divided into two groups $(<26)$ are considered a poor practice and $(>27)$ are considered as good practice.

\section{RESULTS}

In this study, descriptive statistics analysis such as frequencies, percentage, means, and standard deviation was used to assess the socio-demographic characteristics, knowledge, and practice of the respondents, while inferential statistical analysis used to assess is there any relationships between mother's knowledge and practice. The study findings divided into three sections. Section one discussed statistical data analysis procedure by introducing the data management, which included data screening process for cleaning and finding any missing data to define specified data values as user missing to distinguish between two forms of missing data; first, if the respondent refused to answer a question, and second, if the question did not apply to that respondent through SPSS software Ver.22. The response rate of participants was $88 \%$ as there were some cases who declined to answer some specific questions. 
Data were coded, inputted and statistical evaluated through SPSS software, were setting at $5 \%$ level of significant which is (0.05), $P \leq 0.05$ considered significant. Section two provides details on the study findings regarding research questions of the study through descriptive and inferential statistics analysis to determine whether any relationship between the study variables. The final section presented the overall summary of data analysis results of the study. The study findings were conveyed according to the following sections based on the objectives of the study. The mean age of the mother was $26.92 \pm 5.785(n=50)$ with minimum and maximum age 16 and 38 .

Table 1 shows that the characteristic of the sample according to the socio-demographic data, $40 \%$ of the participants of the mothers were between ages 24 and 30 years old, while the minimum mother age groups is $>30$ years old and is 14 participants. Regarding the Educational Levels of mothers, the majority of the participants were in secondary school $(38 \%)$. besides, the lowest percentage $(6 \%)$ were in tertiary (university-college). Moreover, the majority of the occupation status of the mothers was housewife $(90 \%)$, while the lowest of the occupation status of the mothers was students $(2 \%)$.

Regarding monthly Income of Respondent, the result was $42 \%<300,000$ IQD, while the Age of Respondent of the Childs, the majority of the participants is $66 \%$. Their ages were less one year as infant's ages, besides. The lowest ages of the child's (34\%) were between 2 and 3 years. Regarding the gender of respondent of the child's, the majority were in male $(58 \%)$ while the lowest was female (42\%).

As shown in Figure 1, the level of total knowledge of mothers about diarrhea in children under 3 years old was poor knowledge $(68 \%)$. Besides, the level of total knowledge of mothers about diarrhea in children $<3$ years old was good knowledge (32\%).

As shown in Figure 2, the level of total mothers' practice about diarrhea in children under 3 years old was poor practice $(64 \%)$. Besides, the level of mothers practice about diarrhea in children under 3 years old was good practice $(36 \%)$.

\section{Relationship Between Mother's Knowledge and Mother's Practice}

To identify the relationship between the two continuous variables underlying the study, scatterplot was used before observe the present of pattern of linear relationship between the two continuous variables, mother knowledge, and practice as showed in Figure 3.

The result of the research illustrated that there is no association between mother knowledge and mother
Table 1: Socio-demographic characteristics of respondents at the baseline $(n=50)$

\begin{tabular}{lc}
\hline Variables & $n(\%)$ \\
\hline Mothers age (Year) & $16(32)$ \\
$<23$ & $20(40)$ \\
$24-30$ & $14(28)$ \\
$>30$ & \\
Educational level & $16(32)$ \\
$\quad$ Not educated & $12(24)$ \\
Primary school & $19(38)$ \\
Secondary school & $3(6)$ \\
Tertiary (University-College) & \\
Occupation status & $45(90)$ \\
Housewife & $1(2)$ \\
Student & $4(8)$ \\
Employee & \\
Monthly income (IQD*) & $21(42)$ \\
$<300.000$ & $19(38)$ \\
300.000-600.000 & $10(20)$ \\
$>600.000$ & \\
Child age (Year) & $33(66)$ \\
$\quad \leq 1$ years & $17(34)$ \\
2-3 years & \\
Gender (Child) & $29(58)$ \\
Male & $21(42)$ \\
Female & $50(100)$ \\
Total & \\
(IQD*) Iraqi Dinars &
\end{tabular}

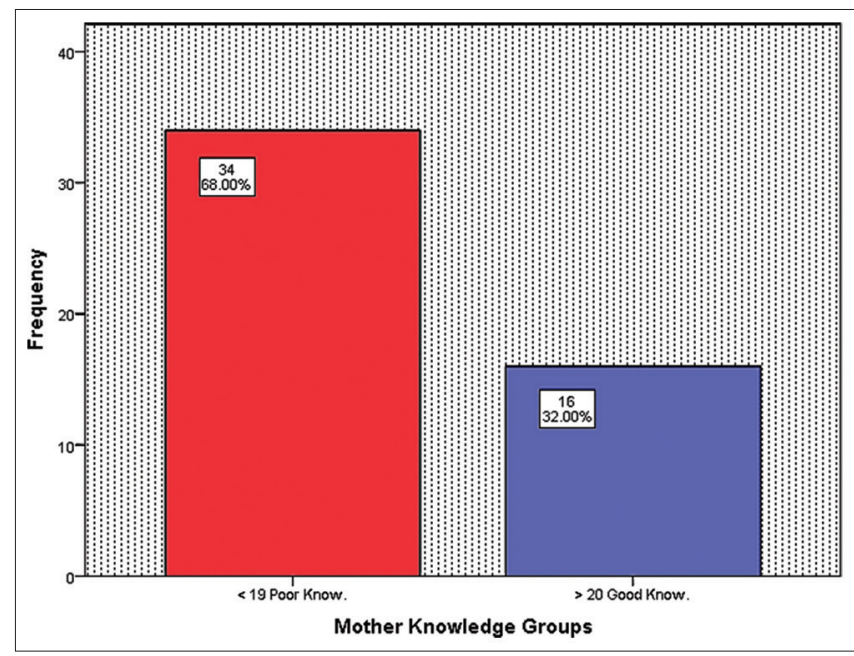

Figure 1: Mother knowledge groups of the respondent $(n=50)$

practice the $r$-value score is $r=-0.458$, which is more than $3, P<0.001$. Thus, there is a negative association between mother knowledge and mother practice Moreover, scores recorded as categorical variables mother knowledge into groups, poor knowledge $(<19)$; and good knowledge $(>20)$, while the divided into groups poor mother practice $(<26)$, and good mother practice $(<27)$. The Bivariate correlations - Pearson test was used as its recommended to understand the association between two quantitative variables. 


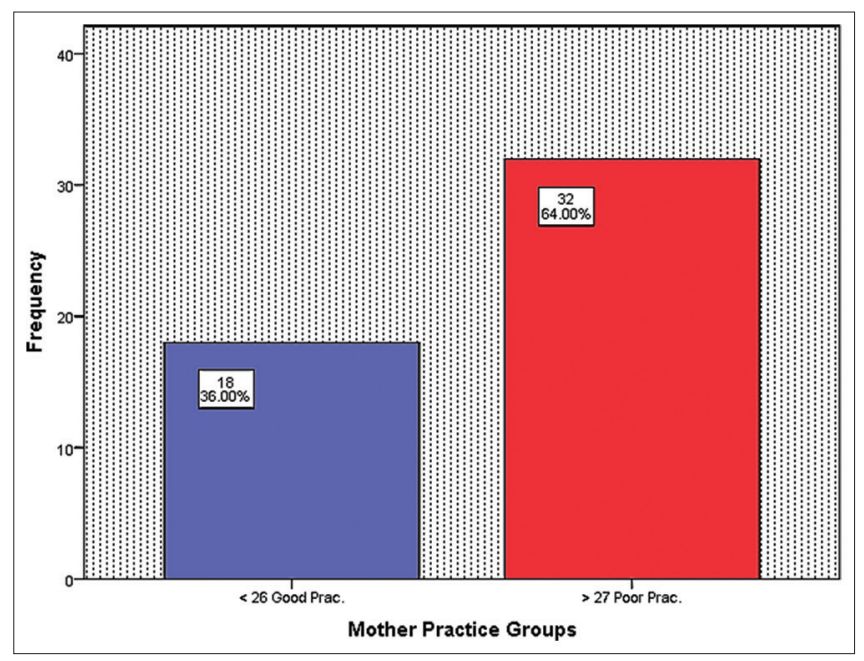

Figure 2: Mother practice groups of the respondent $(n=50)$

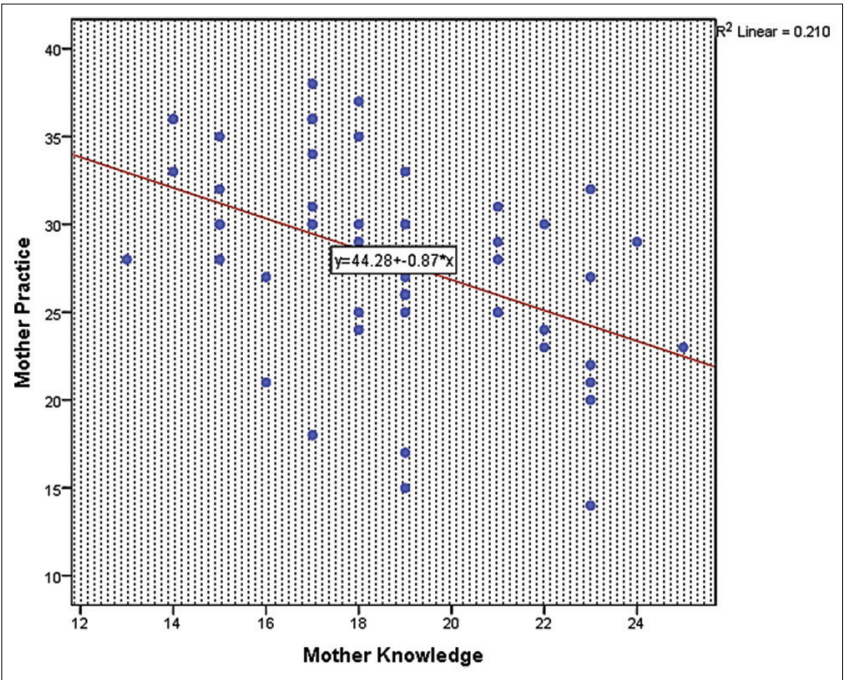

Figure 3: Scatterplots relationship between mother knowledge and practice

\section{DISCUSSION}

In general, the results shown that socio-demographic characteristics such as mother educational, monthly income, and occupation have a role in presenting diarrhea among children $<3$ years old and it affects the knowledge and practice of mothers to deal with diarrhea. As well as, shown that the knowledge and practice of mothers have a negative relationship with presenting diarrhea and knowledge and practice of mother are related to each other (Abu-Baker, et al., 2013).

Childhood diarrhea is a widespread problem in most countries and is a common public health concern. In Kurdistan Region of Iraq, where the study was conducted, there is an increasing incidence of diarrhea in children under 3 years old. Despite the increasing incidence of diarrhea in Iraq and common public concern, there are several discrepancies about the knowledge and practice of diarrhea among the mothers. In general, socio-demographic factors such as mothers' education and occupation, and mother's income are linked with mothers' knowledge about diarrhea and its practice.

The result of this study showed that the majority of the participants was in secondary educational levels (38\%) with recording a lowest percentage $(6 \%)$ were in tertiary (universitycollege). Regarding to the mother's knowledge (68\%) was poor knowledge, as well $(32 \%)$ mothers' knowledge about diarrhea in children $<3$ years old was good knowledge, this is relatively lower than the findings from the similar study conducted in Pakistan (Shah et al., 2015). This difference from may be due to that mothers or may have been provided more information about diarrhea in the study area.

The study showed that only $36 \%$ of mothers had good practice about diarrhea. Moreover, $64 \%$ were poor practice. However, the opposite was observed in studies conducted in Pakistan (Shah et al., 2015).

\section{CONCLUSIONS}

In the action's that mothers were taken, the minimum required is a brief and superficial examination of the dehydrated child and the type and amount of liquid feed to him/her in the case of diarrhea; however, these steps and actions are very important for pediatric welfare. In conclusion, these findings indicate that due to lack of knowledge and poor practice about management of diarrhea the children were suffering from many complications.

\section{RECOMMENDATIONS}

There is need to do more studies to aware mothers of children $<3$ years of age about the management of diarrhea at home to decrease the morbidity and mortality among children less than 3 years old.

Health education should be provided for the mothers on preparation of ORS, home fluid preparation, prevention of diarrhea, and sign of dehydration. Providing health information through mass media and giving awareness section to clarify public view about the significance of nutrition during diarrheal episodes. In addition, this health information is important for physical growth, intellectual growth, and development of children.

\section{REFERENCES}

Abu-Baker, N. N., H. F. Gharaibeh, H. M. Al-Zoubi, C. Savage and M. K. Gharaibeh. 2013. Mothers' knowledge and practices of managing minor illnesses of children under five years. J. Res. 
Nurs. 18(7): 651-666.

Agegnehu, M. D., L. Bewket Zeleke, Y. A. Goshu, Y. L. Ortibo and Y. M. Adinew. 2019. Diarrhea prevention practice and associated factors among caregivers of under-five children in enemay district, Northwest Ethiopia. J. Environ. Public Health. 2019: 5490716

Ahs, J. W., W. Tao, J. Löfgren and B. C. Forsberg. 2010. Diarrheal diseases in low-and middle-income countries: Incidence, prevention and management. Open Infect. Dis. J. 4(1): 113-124.

Al-Hindi, A. I. 2017. Epidemiology of acute diarrheal diseases among Children under five in Gaza Strip: Post war community based study. Sch. J. Appl. Med Sci. 5(2B): 442-452.

Asghar, I., A. Javaid, M. Anwar and G. Mustafa. 2016. Knowledge and practices of rural mothers about diarrhea among infants. J. Sheikh Zayed Med. Coll. 7(4): 1053-1056.

Badowski, N., C. M. Castro, M. Montgomery, A. J. Pickering, S. Mamuya and J. Davis. 2011. Understanding household behavioral risk factors for diarrheal disease in Dar es Salaam: A photovoice community assessment. J. Environ. Public Health, 2011: 130467.

Banik, P. 2013. A Study to Evaluate the Effectiveness of Video Assisted Teaching Programme on Knowledge and Practice Regarding Prevention and Home Management of Dehydration among the Mothers of Under Five Children in a Selected Rural Area of Bangalore (Doctoral Dissertation).

Blaabjerg, S., D. M. Artzi and R. Aabenhus. 2017. Probiotics for the prevention of antibiotic-associated diarrhea in outpatients, a systematic review and meta-analysis. Antibiotics. 6(4): 21.

Chandra, R. K. 2012. Departments of pediatrics and medicine. Antioxid. Nutr. Immune Funct. 262: 13.

Chavan, S. C., S. Agarkhedkar, D. S. Chavan, R. P. Nagdawane and S. Singhania. 2013. Prevalence of rotavirus diarrhoea among children hospitalized in a tertiary care hospital in Western India. Int. J. Pharm. Biomed. Sci., 4(1): 4-7.

Di Genova, B. M. and R. R. Tonelli. 2016. Infection strategies of intestinal parasite pathogens and host cell responses. Front. Microbiol. 7: 256.

Grimason, A. M., S. J. Masangwi, T. D. Morse, G. C. Jabu, T. K. Beattie, S. E. Taulo and K. Lungu. 2014. Knowledge, awareness and practice of the importance of hand-washing amongst children attending state run primary schools in rural Malawi. Int. J. Environ. Health Res. 24(1): 31-43.

Hamed, E. A. E. 2016. Knowledge, Attitude and Practice of Mothers Regarding Diarrheal Disease in Under Five Years Children in Wad Adam Village, Algorashy Locality, Gezira State, Sudan, 2015. Doctoral dissertation, University of Gezira, Gezira.

Hussein, S. H. 2018. Risk Factors of Diarrhea Disease among Under Five in Ibrahim Malik Teaching Hospital, Khartoum state-Sudan, 2017. Doctoral Dissertation, University of Gezira, Gezira.

Islam, M. M., M. Ibrahim, I. J. Ira, M. A. Torab, M. Z. Hossen and Z. Hossain. 2018. A review on health and nutrition status in Bangladesh: Issues and challenges. Int. J. Biomed. Eng. Clin. Sci. 4(2): 48.

Mahor, G. R. 2013. Knowledge and attitudes of mothers regarding use of oral rehydration solution in management of diarrhea. Asian J. Biomed. Pharm. Sci. 3(22): 6-8.

Mathur, A., D. Baghel, J. Jaat, V. Diwan and A. Pathak. 2019.
Community-based participatory research and drug utilization research to improve childhood diarrhea case management in Ujjain, India: A cross-sectional survey. Int. J. Environ. Res. Public Health. 16(9): 1646.

Matsebula-Myeni, Z. 2019. Investigating the Effect of Interventional Programmes in Combatting Inappropriate Use of Antibiotics in Managing and Treating Acute Gastroenteritis in Children Younger than Five Years at the Raleigh Fitkin Memorial Hospital in ESwatini. Doctoral Dissertation, Stellenbosch University, Stellenbosch.

Mokomane, M., I. Kasvosve, E. D. Melo, J. M. Pernica and D. M. Goldfarb. 2018. The global problem of childhood diarrhoeal diseases: Emerging strategies in prevention and management. Ther. Adv. Infect. Dis. 5(1): 29-43.

Munos, M. K. C. L. F. Walker and R. E. Black. 2010. The effect of rotavirus vaccine on diarrhoea mortality. Int. J. Epidemiol. 39(1): i56-i62.

O'Ryan, M., V. Prado and L. K. Pickering. 2005, April. A millennium update on pediatric diarrheal illness in the developing world. In: Seminars in Pediatric Infectious Diseases. Vol. 16. WB Saunders, Philadelphia, PA. p125-136.

Shah, I., S. Arif and A. Ilyas. 2015. Mother's knowledge and attitude associated with diarrhea in an Urban Area in Karachi, Pakistan. Int. J. Innov. Res. Dev. 4(5): 2278-2211.

Shane, A. L., R. K. Mody, J. A. Crump, P. I. Tarr, T. S. Steiner, K. Kotloff, J. M. Langley, C. Wanke, C. A. Warren, A. C. Cheng and J. Cantey. 2017. 2017 infectious diseases society of America clinical practice guidelines for the diagnosis and management of infectious diarrhea. Clin. Infect. Dis. 65(12): e45-e80.

Singh, A., V. Kumar, S. Panesar, M. Adhikary, A. Das and S. Dixit. 2019. Epidemiological investigation of acute gastroenteritis in a residential campus area of Delhi, India. Epidemiol. Int. 4(2): 34-38.

Smith, J. L. and P. M. Fratamico. 1995. Factors involved in the emergence and persistence of food-borne diseases. J. Food Prot. 58(6): 696-708.

Spika, J. S., J. E. Parsons, D. Nordenberg, J. G. Wells, R. A. Gunn and P. A. Blake. 1986. Hemolytic uremic syndrome and diarrhea associated with Escherichia coli 0157: H7 in a day care center. J. Pediatr. 109(2): 287-291.

Suliman, S. S. M. 2017. Knowledge, Attitude, and Practices of Mother of under Five Children toward Home Management of Diarrhea in Wad alfadni Village, El Hassahiesa Locality, Gezira State, Sudan, 2016. Doctoral dissertation, University of Gezira, Gezira.

Sumampouw, O. J., J. E. Nelwan and A. A. Rumayar. 2019. Socioeconomic factors associated with diarrhea among underfive children in Manado Coastal Area, Indonesia. J. Glob. Infect. Dis. 11(4): 140.

Teshale, E. H., S. P. Grytdal, C. Howard, V. Barry, S. Kamili, J. Drobeniuc, V. R. Hill, S. Okware, D. J. Hu and S. D. Holmberg. 2010. Evidence of person-to-person transmission of hepatitis $E$ virus during a large outbreak in Northern Uganda. Clin. Infect. Dis. 50(7): 1006-1010.

WHO, and UNICEF. 2015a. Water, Sanitation and Hygiene in Health Care Facilities: Status in Low-and Middle-income Countries and Way Forward. World Health Organization, Geneva. Available from: http:// www.apps.who.int/iris/bitstream/10665/154588/1/9789241508476_ eng.pdf?ua=1. [Last accessed on 2015 Oct]. 